\title{
Research on Camera Calibration of the Binocular Vision Light Pen Measurement System
}

\author{
Feng shou Zhang ${ }^{1,}$, Jian li Jin $^{2, b}$ \\ ${ }^{1}$ School of Medical Technology and Engineering, Henan University of Science and Technology, \\ Luoyang 471003, China; \\ ${ }^{2}$ School of Mechatronics and Engineering, Luo Yang 471003,China. \\ afengshouzhang@163.com, ${ }^{\mathrm{b}}$ hauster @163.com
}

Keywords: Camera calibration, Lens distortion, Plane templates calibration method, Coded identification.

\begin{abstract}
To binocular vision light pen measuring system, calibrating the camera fast and accurate, can improve the practicality of the system application. In the establishment of ideal camera imaging model, considering the lens radial distortion and tangential distortion factors, studies the practical imaging model parameters. Plane template calibration algorithm is improved. Using points which are near calibration template center and distortion smaller solve the initial model parameters. Application internal mapping Newton's method of subspace confidence domain method solves global optimization model parameters. Designing plane calibration template and using coded identification method, identify the signs point in the calibration template. The calibration efficiency is improved. The experiments show that the calibration method can be achieved binocular stereo vision camera calibration.
\end{abstract}

\section{Introduction}

Binocular vision, light pen measuring system is based on using two cameras to imaging, and through a series of coordinate transformation relationship to determine the spatial position and posture of light pen and then get space coordinate of measuring points. The mutual mapping relationship between space three-dimensional coordinates of the object surface some point and its corresponding $2 \mathrm{~d}$ coordinates in the image is determined by the camera imaging model. In general, the camera model parameters can be obtained through experiment and optimization calculation, and the process of solving camera model geometry and optical parameters is called camera calibration. At present, there is no universal calibration method that can be used for all kinds of situation, if the specific applications are different and the calibration method is also different. Here is a commonly used calibration method.

Direct linear transformation (DLT) method [1], its principle is simple and easy to implement, But the disadvantage is that calibration precision is not high and intermediate parameter has constraint each other. moreover, it is not easy to separate internal parameter values from external parameter values. In order to improve the calibration accuracy further, based on the DLT method, Tsai [2] first proposed two-step method based on radial alignment constraint (RAC), which has higher precision and is suitable for precision measurement, but only considering the radial distortion, it does not apply to simple calibration. In order to improve the calibration of flexibility, Zhang Zhengyou [3] first proposed plane template calibration method, which can obtain internal and external parameter values for the camera model by making the camera collect more than three images of the calibration board from different angles and use information of the known feature point location on the template and correspondence of the corresponding projection points in the image, because the calibration is simple, it is widely used. But because of only considering the radial distortion, it is not applicable with large distortion fisheye lens. In addition, camera self-calibration method [4-6] and the calibration method based on active vision [7].which have low calibration precision and complex calibration process, is not suitable for optical measurement system. 
We adopt the improved plane model method for two camera calibration aiming at Light pen for binocular vision measurement system in this paper. For the calibration method in this paper, the camera radial distortion and tangential distortion are considered, calibrating part points of template center near is adopted in the solution process of model parameters' initial value, which improves the accuracy of the parameter's initial value. For actual imaging model considering the distortion, all points on the template should be used to do the optimization algorithm for precise. At the same time, in order to improve the efficiency of calibration, the coding landmark to identify all the feature points on the calibration template is used.

\section{Modeling of the camera}

Ideal model of the camera. The camera mode can be obtained through the simple center Perspective projection and rigid body rotation and transform translation. This model is linear, not considering the lens distortion, which is as shown in Fig.1.

In the camera model, $O_{w}, X_{w}, Y_{w}, Z_{w}$ is world coordinate system. $O_{c}, X_{c}, Y_{c}, Z_{c}$ is the camera coordinate system, the $O_{c}$ is the camera lens optical center, $X_{c}, Y_{c}$ axis is parallel to the imaging plane, $Z c$ axis is perpendicular to the imaging plane the intersection between them is $\left(u_{0}, v_{0}\right)$, which is the main point of the camera ,namely $O_{I} . O_{c} O_{I}$ is the focal length f. $O_{I}, x y$ is the image plane coordinate system, where the $\mathrm{x}$ axis and y axis are parallel to $X_{c}, Y_{c}$ shaft axis respectively, $O_{I}$ is the intersection point of the Zc shaft and CCD. $O_{0}, u, v$ is the image plane coordinate system of computer, where the origin of coordinates $O_{0}$ is selected in the top left corner of the image, the $u$ axis is to the right and parallel to the $X$ axis, while the u Shaft is downward and parallel to the $Y$ axis.

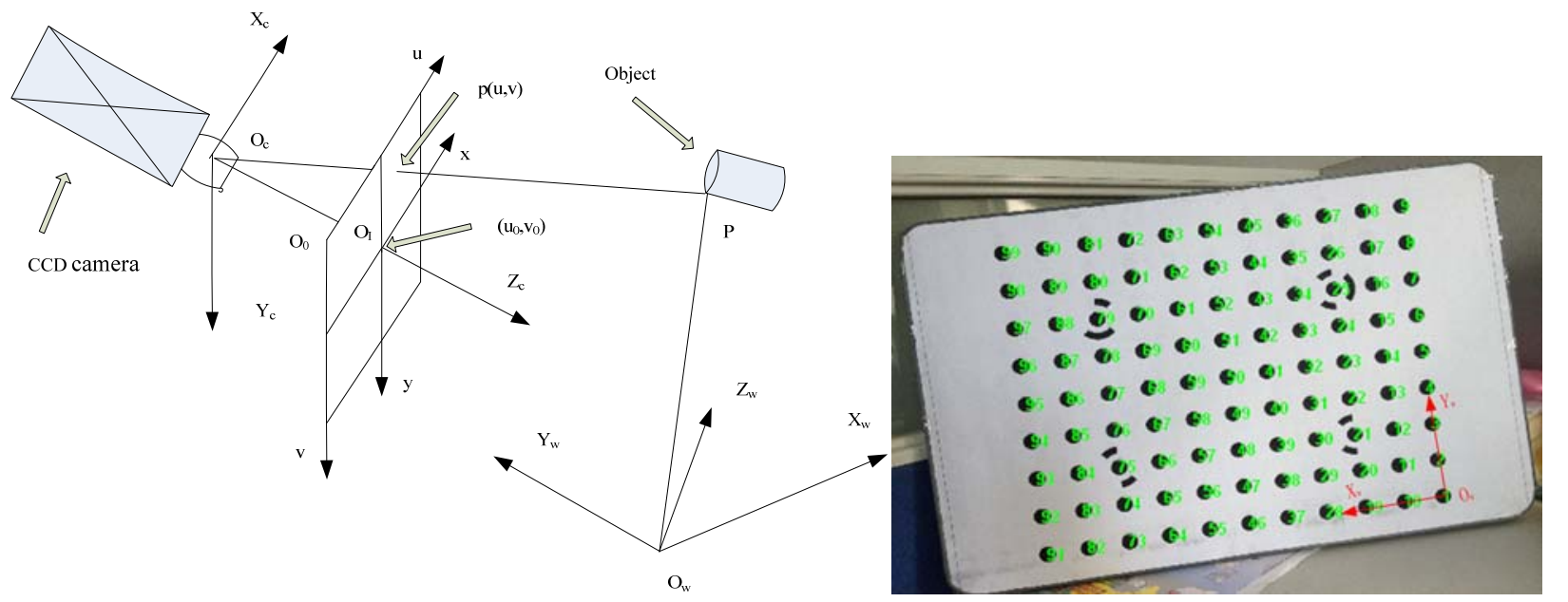

Fig.1 Ideal model of the camera

Fig.2 The serial number of all marking point in the plane calibration template

By camera model between different coordinate system transformation relations, can get the corresponding relations between any Point $\left(X_{w}, Y_{w}, Z_{w}\right)$ of the space and its projection point $\mathrm{P}(u, v)$ in the image:

where, $A=\left[\begin{array}{ccc}s_{x} f / d x & \mu f / d x & u_{0} \\ 0 & f / d y & v_{0} \\ 0 & 0 & 1\end{array}\right], M=[R T]$.In the Matrix $A$, make $\alpha=s_{x} f / d x, \beta=f / d y$ which

$$
Z_{c}\left[\begin{array}{c}
u \\
v \\
1
\end{array}\right]=A M\left[\begin{array}{c}
X_{w} \\
Y_{w} \\
Z_{w} \\
1
\end{array}\right]
$$


respectively represent scaling factor of $u$ axis and $V$ axis, $\gamma=\mu f / d x$ describes inclination angles of the two shaft, the Matrix A can be abbreviated as $A=\left[\begin{array}{ccc}\alpha & \gamma & u_{0} \\ 0 & \beta & v_{0} \\ 0 & 0 & 1\end{array}\right]$, which be called the camera

parameters matrix, is Only related to the camera internal parameters, which contains such basic camera internal parameters as scale factor, focal length, inclination factor, as well as the main point representing the characterization of the geometric and optical properties of the camera, including the parameters for calibration. $M$, which contains an $3 \times 3$ orthogonal rotation matrix $R$ and $3 \times 1$ translation matrix $T$, is the external parameters matrix of camera, respectively defining the attitude and position of machine beams in the world coordinate system.

The actual camera model containing lens distortion. There exist all kinds of distortions, resulting in position error between actual image point on the space object and the ideal image point. Experiments show that the farther from the center of a image the position is, the worse distortion is, describing the nonlinear model of the camera usually adopts the following Eq.(2).

$$
\left\{\begin{array}{l}
x_{d}=x+\delta_{x}(x, y) \\
y_{d}=y+\delta_{y}(x, y)
\end{array}\right.
$$

where the $\left(x_{d}, y_{d}\right)$ is actual image coordinates; the $(x, y)$ - the ideal image coordinates.

Generally, camera contains radial distortion and tangential distortion, centrifugal distortion and thin prism distortion, etc. the distortion can be compensated through the radial distortion and tangential distortion. The general distortion model can be represented :

$$
\left\{\begin{array}{l}
\delta_{x}(x, y)=\delta_{x r}+\delta_{x t}=x\left(k_{1} r^{2}+k_{2} r^{4}+k_{3} r^{6}+\cdots\right)+2 p_{1} x y+p_{2}\left(r^{2}+2 x^{2}\right) \\
\delta_{y}(x, y)=\delta_{y r}+\delta_{y t}=y\left(k_{1} r^{2}+k_{2} r^{4}+k_{3} r^{6}+\cdots\right)+2 p_{2} x y+p_{1}\left(r^{2}+2 y^{2}\right)
\end{array}\right.
$$

where $k_{1}, k_{2}, k_{3}$ are radial distortion coefficient; $p_{1}, p_{2}$ are tangential distortion coefficient; (x,y) is ideal image coordinates; $r^{2}=x^{2}+y^{2}$.

Combining Eq. (2) with Eq. (3), we can get the corresponding actual image coordinates of space point. After taking into account the lens distortion, the actual camera imaging model becomes nonlinear model and the parameters need to be solved by the nonlinear model.

\section{Solving parameters of camera model}

The solution of projection matrix $\mathbf{H}$. Assuming planar calibration board in the world coordinate system $X_{w} Y_{w}$, there will always be $Z_{w}=0$. (1) can be written as

$$
s\left[\begin{array}{c}
u \\
v \\
1
\end{array}\right]=A\left[\begin{array}{llll}
r_{1} & r_{2} & r_{3} & t
\end{array}\right]\left[\begin{array}{c}
X_{w} \\
Y_{w} \\
0 \\
1
\end{array}\right]=A\left[\begin{array}{lll}
r_{1} & r_{2} & 1
\end{array}\right]\left[\begin{array}{c}
X_{w} \\
Y_{w} \\
1
\end{array}\right]=H\left[\begin{array}{c}
X_{w} \\
Y_{w} \\
1
\end{array}\right]
$$

where $r_{1}, \quad r_{2}$ and $r_{3}-1,2$ and 3 column vector of rotation matrix R; $H=A\left[\begin{array}{lll}r_{1} & r_{2} & t\end{array}\right]$ is called as the projection matrix.

Clearly, $\mathrm{H}$ is a $3 \times 3$ matrix defining on a scale factor. If known $M$ on the planar calibration board and projection matrix $H$ are known at the same time, you can use Eq.(4) to calculate M's corresponding projection point $\mathrm{m}$ on the image plane. Ideally, a point $\mathrm{M}$ on the planar calibration board and its corresponding image points in the plane should satisfy Eq. (4). But actually, center coordinates of landmark point extracted by from image processing exists interference noise, and this leads that $M_{i}$ and $m_{i}$ can't strictly satisfy Eq. (4). Calculation of projection matrix $\mathrm{H}$ is converted 
into the process of making residuals minimum between actual image coordinates $m_{i}$ and the image coordinates $\hat{m}_{i}$ calculated from Eq. (4), the objective function is

$$
\min \sum_{i}\left\|m_{i}-\hat{m}_{i}\right\|^{2}
$$

If we want to solve the type, an initial value needs to be known and we can get it using a direct linear transformation (DLT) model.

The camera's internal and external parameter initial value solution. Make $H=\left[h_{1}, h_{2}, h_{3}\right]$, we can get:

$$
\left[h_{1}, h_{2}, h_{3}\right]=\lambda A\left[\begin{array}{lll}
r_{1} & r_{2} & t
\end{array}\right]
$$

Where $\lambda$ - any scaling factor; $r_{1}$ and $r_{2}$--Orthogonal unit vector, then $r_{1}^{T} r_{2}=0 \quad r_{1}^{T} r_{1}=I=r_{2}^{T} r_{2}$ Thus there is:

where $A^{-T}=\left(A^{-1}\right)^{T}=\left(A^{-T}\right)^{-1}$.

$$
\left\{\begin{array}{c}
h_{1}^{T} A^{-T} A^{-1} h_{2}=0 \\
h_{1}^{T} A^{-T} A^{-1} h_{1}=h_{2}^{T} A^{-T} A^{-1} h_{2}
\end{array}\right.
$$

Make matrix $B=\lambda\left(A^{-1}\right)^{T} A^{-1}, \lambda$ is an arbitrary scaling factor, because $A$ is triangular matrix and reversible, we can know that $B$ is the symmetric matrix. We use $6 \mathrm{~d}$ vector to describe it as

Thus, available

$$
b=\left[\begin{array}{llllll}
B_{11} & B_{12} & B_{22} & B_{13} & B_{23} & B_{33}
\end{array}\right]^{T}
$$

$$
h_{i}^{T} B h_{j}=v_{i j}^{T} b
$$

where $h_{i}=\left(h_{1 i}, h_{2 i}, h_{3 i}\right)^{T}$ is the first column of the projection matrix; $\mathrm{v}_{\mathrm{ij}}=\left[\mathrm{h}_{1 \mathrm{i}} \mathrm{h}_{1 \mathrm{j}} \mathrm{h}_{1 \mathrm{i}} \mathrm{h}_{2 \mathrm{j}}+\mathrm{h}_{2 \mathrm{i}} \mathrm{h}_{1 \mathrm{j}} \mathrm{h}_{2 \mathrm{i}} \mathrm{h}_{2 \mathrm{j}}\right.$ $\left.\mathrm{h}_{3 \mathrm{i}} \mathrm{h}_{1 \mathrm{j}}+\mathrm{h}_{1 \mathrm{i}} \mathrm{h}_{3 \mathrm{j}} \mathrm{h}_{3 \mathrm{i}} \mathrm{h}_{2 \mathrm{j}}+\mathrm{h}_{2 \mathrm{i}} \mathrm{h}_{3 \mathrm{j}} \mathrm{h}_{3 \mathrm{i}} \mathrm{h}_{3 \mathrm{j}}\right]^{\mathrm{T}}$ 。

For every planar calibration board image, two constraints can be obtained represented by Eq. (7), it is written in matrix form

$$
\left[\begin{array}{c}
v_{12}^{T} \\
\left(v_{11}-v_{22}\right)^{T}
\end{array}\right] b=0
$$

If there are $n$ pieces of different position images of the plane calibration template, $n$ related expression can be formed, there is

$$
V b=0
$$

Yes $\mathrm{V}$ is $2 n \times 6$ matrix type, which is obtained from the projection matrix.

Solution of Eq. (11) is the eigenvectors correspond to the smallest eigenvalue of matrix $V^{T} V$. Because $b$ is a 6-dimension column vector, then, at that time, $n \geq 3$ the equation has a unique solution or solving overdetermined equation can obtain an optimal solution. So, when calibrated, images of the planar calibration board shall be collected at least three different positions and any two images can not be parallel. After solving $b, B$ is available then we can get matrix $A$ of the camera internal parameters. Thus, we can get the camera external parameters

$$
\left\{\begin{array}{l}
r_{1}=\kappa A^{-1} h_{1} \\
r_{2}=\kappa A^{-1} h_{2} \\
r_{3}=r_{1} \times r_{2} \\
t=\kappa A^{-1} h_{3}
\end{array}\right.
$$

where $\kappa=\frac{1}{\left\|A^{-1} h_{1}\right\|}=\frac{1}{\left\|A^{-1} h_{2}\right\|}, r_{i}$ is the $\mathrm{i}(i=1,2,3)$ column of the rotation matrix. 
Because of the noise, $R=\left[\begin{array}{lll}r_{1} & r_{2} & r_{3}\end{array}\right]$ can not satisfy the orthogonality and the optimization calculation is required. Considering that distortion of near point of image center is small, this does not need to be considered, optimization guidelines can be built depending on the ideal perspective model, which is as follows:

$$
\min =\sum_{i=1}^{n} \sum_{j=1}^{p_{i}}\left\|m_{i j}-\hat{m}\left(A, R_{i}, t_{i}, M_{i j}\right)\right\|^{2}
$$

Constraints should satisfy the orthogonality, the objective of optimization is to solve all of the parameters of $A, R_{i}, t_{i}$ further. $p_{i}$ signify the number of the sign points of near the center selected from the first image, $m_{i j}$ is the coordinate of the j point of the actual image in the i image $M_{i j}$ is the corresponding point of the plane calibration template, $\hat{m}\left(A, R_{i}, t_{i}, M_{i j}\right)$ is the image coordinates point which needs to put $M_{i j}$ Eq. (4) and calculate it.In theory, and the it is better that $m_{i j}$ is closer to $\hat{m}\left(A, R_{i}, t_{i}, M_{i j}\right)$. Use the gradient descent method to do optimize calculation, because initial value algorithcm can quickly converge accurately.

All Parameters solution of a camera containing optical distortion. In order to get more precise camera parameters, all of the world coordinates and corresponding image points are need to be taken into consideration。After considering the camera radial distortion and tangential distortion ,the optimization guideline is

$$
\min =\sum_{i=1}^{n} \sum_{j=1}^{p}\left\|m_{i j}-\hat{m}\left(k_{1}, k_{2}, k_{3}, p_{1}, p_{2}, A, R_{i}, t_{i}, M_{i j}\right)\right\|^{2}
$$

Constraints are that all $R_{i}$ should satisfy the orthogonality, the purpose of optimization model is to accurately solve the $k_{1}, k_{2}, k_{3}, p_{1}, p_{2}, A, R_{i}, t_{i}, M_{i j}$. Among them, $p$ represents all the marked points selected from the i image $k_{1}, k_{2}, k_{3}, p_{1}, p_{2}$ is the distortion coefficient, because its value is generally small, initial value can be set to zero. $\hat{m}\left(k_{1}, k_{2}, k_{3}, p_{1}, p_{2}, A, R_{i}, t_{i}, M_{i j}\right)$ is the actual image coordinate points calculated by $M_{i j}$, definition of other parameters is as shown in the type (13). Using initial value of model parameters obtained in the first step and subspace confidence domain method based on the internal mapping Newton's method, we can solve the optimization model Eq. (14).

\section{Calibration of pairs of camera}

A rotation matrix $R$ and translation matrix $T$ can be used to represent the relative position between the two cameras; the location relationship between the world coordinate system and left the camera is represented by the rotation matrix $R_{r}$ and translation matrix $T_{r}$; So, to the world coordinates of a point $P_{w}\left(x_{w}, y_{w}, z_{w}\right)$, there is

$$
\left\{\begin{array}{l}
p_{c l}=R_{l} P_{w}+T_{l} \\
p_{c r}=R_{r} P_{w}+T_{r}
\end{array}\right.
$$

where $p_{c l}$ and $p_{c r}$ - the corresponding point of world coordinate system $P_{w}$ point in the camera coordinate system. Relative position relationship of the two cameras can be obtained from type (15)

$$
\left\{\begin{array}{l}
R=R_{r} R_{1}^{-1} \\
T=T_{r}-R_{r} R_{l}^{-1} T_{l}
\end{array}\right.
$$

\section{Camera calibration template.}

Because diagonal point extraction accuracy in the current image processing is not high and the checkerboard and latticed logo is hard to realize encoding recognition, this article uniformly uses the circular logo as the calibration template primitives, as is shown in Fig.2 .Calibration template consists 
of $9 \times 11$ black solid circles with white background, there are four solid circular ring adopted encoding [7]. The distance between the adjacent signalized points' circle centers is $40 \mathrm{~mm}$ in calibration template. Image plane center subpixel coordinates of filled circle in calibration template can be obtained by using image processing techniques and ellipse fitting method. When recognizing numbered of the landmark in calibration template the image, firstly, four encoding landmark of template is obtained by image processing technology in and encoding value is obtained according to the principle of encoding, meanwhile match the extracted pixel center of the encoding image with its code value. Then, recognize the sort of the rest landmarks of the calibration template by using four encoding points and number identification results are shown in Fig.2.

Establishing the world coordinate system is as shown in Fig.2, where the origin $O_{w}$ is set on the center of the landmark in the template number 1. $X w, Y w$ axis directions are as shown in Fig.2. When calibrating template, firstly obtain the world coordinates of signalized point according to the design of the calibration plate size, and then match numbered 1 99 landmark image coordinate with the corresponding world coordinates. By using the front algorithm, solve the camera model parameters.

\section{Camera calibration results}

This article calibrates the left and right camera of the binocular stereo vision system and the camera and lens models respectively is BASLERscA1600-14gc and FUJINON HF50HA-B. The calibration results about the camera internal parameters are shown in Tab.1. The measured center point distribution of calibration template about the camera is shown in Fig.3 and Fig.4

Table 1 camera parameter calibration results

\begin{tabular}{|c|c|c|c|c|c|c|c|c|c|c|}
\hline Camera & $\alpha /$ pixel & $\beta /$ pixel & $\gamma /$ pixel & u0/pixel & v0/pixel & $\mathrm{k} 1 / \mathrm{mm}^{-2}$ & $\mathrm{k} 2 / \mathrm{mm}^{-4}$ & $\mathrm{k} 3 / \mathrm{mm}^{-6}$ & $\mathrm{p} 1 / \mathrm{mm}^{-2}$ & $\mathrm{p} 1 / \mathrm{mm}^{-2}$ \\
\hline Left & 3156.674 & 3116.279 & 0 & 865.824 & 617.082 & 0.17521 & -0.12520 & 0.02313 & 0.04394 & 0 \\
\hline Right & 3156.774 & 3116.473 & 0 & 865.953 & 617.259 & 0.16571 & -0.13121 & 0.01683 & 0.03965 & 0 \\
\hline
\end{tabular}

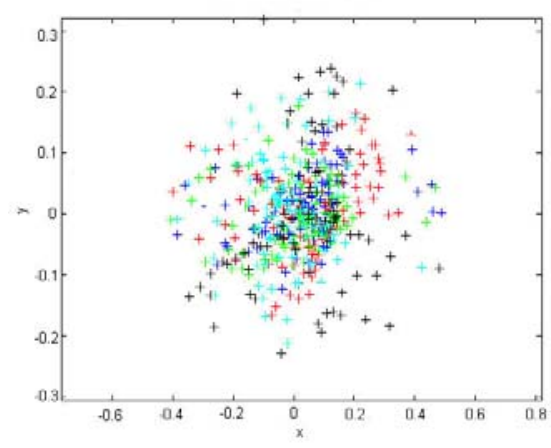

Fig.3 projection Point error distribution of left the camera

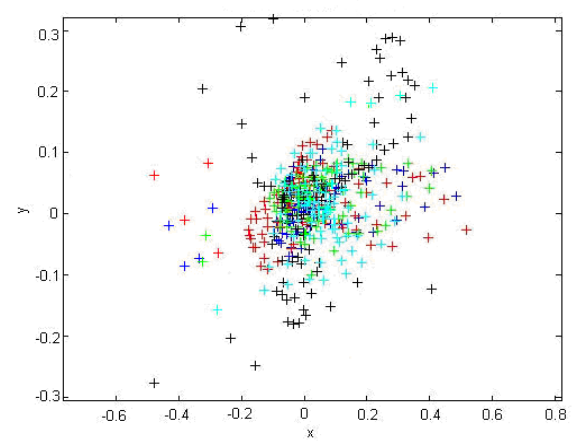

Fig.4 projection Point error distribution of the right camera

After calibrating the camera respectively, get the relative position relations of the two cameras using Eq. (16)

Relative rotation matrix between two cameras:

$$
R=\left[\begin{array}{ccc}
0.9074 & 0.0005 & -0.4238 \\
0.0289 & 0.9995 & -0.0048 \\
0.4285 & -0.0072 & 0.9637
\end{array}\right]
$$

Relative translational matrix between two cameras:

$$
T=\left[\begin{array}{lll}
-358.8084 & -15.8933 & 79.2648
\end{array}\right]^{T}
$$

\section{Conclusion}

Through the establishment of the camera center projection model, give the corresponding relationship between space and its image. Consider radial distortion and tangential distortion of the 
camera. Correct the center projection model. Obtain the practical imaging model of camera and point out of model parameters that are need to be calibrated. Design plane calibration template with function of automatic number identification. Calibrate left and right camera respectively and get the camera's internal parameters.Finally, calibrate the relative position of two camera, lying the foundation for three coordinate measuring of space objects in the measurement system of light pen.

\section{References}

[1]WANG Dong-hong LUO Jun HU Yin-feng. The Method of Direct Liner Transformation on the Camera Calibration [J]. MACHINERY \& ELECTRONICS , 2006, (09): 9-11.

[2]TSAI R Y. A versatile camera calibration technique for high-accuracy 3D machine vision metrology using off-the-shelf tv cameras and lenses. IEEE Journal of Robotics and Automation, 1987, 3(4): 323-344.

[3]ZHANG Z Y. Flexible Camera Calibration by Viewing a Plane from Unknown Orientations. In Proc.7th Intemational Conference on Computer Vision,Kerkyra,Greece,pages:666-673, 1999,9.

[4] LIU Zhen-zhong ,FU Li. Research On camera calibration method [J]. JOURNAL OF SHENYANG INSTITUTE OF AERONAUTICAL ENGINEERING，2010，27(1): 43-47.

[5]Hu Zhanyi, Wu Chaofu. A Review on Some Active Vision Based Camera Calibration Techniques [J]. CHINESE JOURNAL OF COMPUTERS ， 2002， (11): 1149-1156.

[6] WENG J, COHEN P, HENUOU M. Camera calibration with distortion models and accuracy evaluation[J]. IEEE Transactions on Pattern Analysis and Machine Intelligence, 1992, 14(10): 965980.

[7]Fan Hongsheng - Study and application of artificial markers in industrial digital photogrammetry [D]. Zhengzhou: The PLA Information Engineering University, 2006: 16-55. 